Research

Open Access

\title{
Thoracic epidural anesthesia time-dependently modulates pulmonary endothelial dysfunction in septic rats
}

\author{
Stefan Lauer ${ }^{1}$, Hendrik Freise ${ }^{1}$, Martin Westphal ${ }^{1}$, Alexander Zarbock ${ }^{1}$, Manfred Fobker ${ }^{2}$, \\ Hugo K Van Aken ${ }^{1}$, Andreas W Sielenkämper ${ }^{1}$ and Lars G Fischer ${ }^{1}$
}

\begin{abstract}
1Department of Anesthesiology and Intensive Care Medicine, University Hospital Muenster, Albert-Schweitzer-Str. 33,48149 Muenster, Germany ${ }^{2}$ Center for Laboratory Medicine, University Hospital of Muenster, Albert-Schweitzer-Str. 33, 48149 Muenster, Germany

Corresponding author: Stefan Lauer, stlauer@gmx.net

Received: 13 Mar 2009 Revisions requested: 5 May 2009 Revisions received: 2 Jun 2009 Accepted: 6 Jul 2009 Published: 6 Jul 2009

Critical Care 2009, 13:R109 (doi:10.1186/cc7950)

This article is online at: http://ccforum.com/content/13/4/R109

(c) 2009 Lauer et al.; licensee BioMed Central Ltd.

This is an open access article distributed under the terms of the Creative Commons Attribution License (http://creativecommons.org/licenses/by/2.0), which permits unrestricted use, distribution, and reproduction in any medium, provided the original work is properly cited.
\end{abstract}

\begin{abstract}
Introduction Increasing evidence indicates that epidural anesthesia improves postoperative pulmonary function. The underlying mechanisms, however, remain to be determined. Because pulmonary nitric oxide has been identified to play a critical role in pulmonary dysfunction in sepsis, we hypothesized that thoracic epidural anesthesia (TEA) modulates endothelial dysfunction via a nitric oxide-dependent pathway.

Methods Thirty-six Sprague-Dawley rats underwent sham laparotomy or induction of peritoneal sepsis caused by cecal ligation and puncture (CLP). Septic animals were then treated with either bupivacaine $0.5 \%$ or normal saline epidurally $(15 \mu \mathrm{l} /$ $\mathrm{h}^{-1}$ ) for 6 hours or 24 hours after injury. Previous experiments demonstrated that these time points correspond with a hyperdynamic (at 6 hours) and hypodynamic circulation (at 24 hours), respectively. In addition, two sham control groups received either bupivacaine $0.5 \%$ or normal saline epidurally (15 $\left.\mu \mathrm{l} / \mathrm{h}^{-1}\right)$. Six and 24 hours after injury, hemodynamic measurements and arterial blood gas analyses were performed in awake, spontaneously breathing rats. Exhaled nitric oxide, bradykinin-induced pulmonary vasoconstriction (a surrogate marker of endothelial dysfunction), pulmonary wet/dry-weight ratio (an estimate of pulmonary edema), and myeloperoxidase activity (MPO, a surrogate marker of neutrophil infiltration into

lung tisssue) were investigated at 6 and 24 hours by using an established model of isolated and perfused lungs.

Results In hyperdynamic sepsis, treatment with TEA resulted in reduced bradykinin-induced pulmonary vasoconstriction $(P<$ 0.05 ) and lower levels of exhaled NO as compared with those in untreated septic rats $(P<0.05)$. However, the development of pulmonary edema or MPO activity in the lungs was not alleviated by sympathetic blockade in this phase of sepsis. Conversely, TEA led to an increased bradykinin-induced pulmonary vasoconstriction and pulmonary edema despite reduced exNO levels and pulmonary MPO activity in hypodynamic sepsis (each $P<0.05$ versus CLP 24 h). Pulmonary gas exchange was only marginally affected under the influence of TEA in hypodynamic sepsis. Mean arterial pressure and heart rate were not affected beyond the changes caused by sepsis itself.

Conclusions The results of the present study suggest that TEA modulates the NO pathway and exerts positive effects on pulmonary endothelial integrity only in hyperdynamic sepsis. Whether the negative effects on endothelial function in hypodynamic sepsis have an impact on overall morbidity and mortality remains to be determined in future studies.
\end{abstract}

\section{Introduction}

Thoracic epidural analgesia (TEA) is one of the most versatile and widely used neuronal deafferentation techniques to reduce effectively intraoperative sympathetic stimulation resulting from surgical trauma [1]. TEA is therefore an integral part of a multimodal concept to accelerate rehabilitation after major abdominal and thoracic surgery and is increasingly incorporated into therapeutic strategies in critical care medicine [2-4]. Whether TEA is also useful to accelerate the recovery of high-risk patients with severe sepsis and septic shock is still not fully understood.

ABE: actual base excess; BK: bradykinin; CLP: cecal ligation and puncture; exNO: exhaled nitric oxide; iNOS: inducible nitric oxide synthase; MAC: minimum alveolar concentration; MPO: myeloperoxidase activity; NO: nitric oxide; OD: outer diameter; TEA: thoracic epidural anesthesia; VSMC: vascular smooth muscle cell. 
In severe sepsis, pulmonary endothelial dysfunction plays a pivotal role in the development of respiratory failure [5]. In this context, overproduction of nitric oxide (NO) in the lungs has been identified as a major cause for the emergence of endothelial dysfunction and pulmonary edema [6,7]. Likewise, increasing evidence suggests that TEA attenuates pulmonary endothelial injury in ventilated patients after major abdominal surgery. A recently published meta-analysis outlined that TEA reduced the rate of respiratory failure and the duration of required ventilation in those patients [8].

We hypothesized that TEA modulates pulmonary endothelial dysfunction in sepsis via an NO-dependent pathway. The current study was therefore conducted as a prospective, randomized, controlled laboratory experiment to clarify whether and how TEA affects the pulmonary endothelial dysfunction typically seen in the presence of sepsis. Because the early phase of hyperdynamic circulation is typically followed by a late hypodynamic circulatory state in rats with sepsis secondary to cecal ligation and puncture (CLP) [9], another objective of this study was to determine the effects of TEA in the different phases of sepsis. These aims were investigated by using an established model of isolated and perfused rat lungs $[10,11]$.

\section{Materials and methods}

After approval of the District Government, 36 male SpragueDawley rats (Harlan Winkelmann, Borchen, Germany) weighing between 250 and $300 \mathrm{~g}$ were kept in a 12-hour light/dark cycle and had free access to food and water. The animals were included in the study after a 1-week acclimatization period.

\section{Animal preparation}

Anesthesia was induced and maintained by isoflurane inhalation (minimum alveolar concentration (MAC) 1.4 Vol. \%). During instrumentation, spontaneous respiration was maintained. Central venous and arterial lines $(0.96 \mathrm{~mm}$ OD) (Liquiscan, Ueberlingen, Germany) were introduced via the right external jugular vein and the left carotid artery, respectively. Epidural catheters (0.61 mm OD) were inserted at the levels of L3/L4 and advanced to $T 6$. A repeated negative liquid-aspiration test excluded subdural position of the catheter tip. All catheters were protected by a swivel device. After completion of the experimental protocol, the position of the catheter was verified in each animal by autopsy. This model of continuous thoracic epidural analgesia in permanently instrumented awake rats has been shown consistently to produce stable thoracic sympathetic blockade while maintaining hemodynamic stability [12-14].

A midline laparotomy incision was made, and the cecum was ligated just distal to the ileocecal valve so that continuity was preserved. The cecum was then punctured twice with an 18gauge needle. Thereafter, the bowel was returned to the peri- toneal cavity, and the wound was closed in two layers. Rats were then allowed to awaken. Fluid resuscitation with $\mathrm{NaCl}$ $0.9 \%\left(6 \mathrm{ml} / \mathrm{kg}^{-1} / \mathrm{h}^{-1}\right)$ was started postoperatively and continued throughout the in vivo experiment until the aorta was ligated for the isolation and perfusion of the lungs. Fentanyl (Janssen Cilag GmbH, Neuss, Germany; $2 \mu \mathrm{g} / 100^{-1} \mathrm{~g} / \mathrm{h}^{-1}$ ) was continuously infused to ensure adequate analgesia [10].

\section{Experimental protocol}

Because previous experiments demonstrated that CLP sepsis corresponds with a hyperdynamic ( 2 to 10 hours after CLP) and hypodynamic circulation (later than 18 hours after CLP) with a transition from a hyperdynamic to a hypodynamic circulation between 10 and 20 hours, the effects of TEA on pulmonary endothelial dysfunction were determined at 6 and 24 hours, respectively, after CLP $[15,16]$. Taking into consideration that endothelial integrity did not differ between 6 and 24 hours in sham animals in previous studies [17], sham-operated animals were investigated at 24 hours only.

After the instrumentation, rats were randomly divided into six groups ( $n=6$ each) by using a sealed and numbered envelope system. To ensure that the investigator was blinded to the study-group assignment, a technical assistant conducted the laparotomy procedure and the preparation of the epidural infusion. The SHAM group received a sham laparotomy followed by continuous TEA with normal saline $\left(15 \mu \mathrm{l} / \mathrm{h}^{-1}\right.$ for 24 hours, SHAM); the SHAM + TEA group underwent sham laparotomy followed by continuous TEA with isobaric bupivacaine $0.5 \%$ (15 $\mu \mathrm{l} / \mathrm{h}^{-1}$ for 24 hours, SHAM + TEA). In the hyperdynamic sepsis group (6 hours after injury), normal saline $\left(15 \mu \mathrm{l} / \mathrm{h}^{-1}\right)$ was continuously administered epidurally for 6 hours after induction of CLP sepsis (CLP 6 h). The TEA-treated hyperdynamic sepsis group received CLP + continuous TEA with isobaric bupivacaine $0.5 \%\left(15 \mu \mathrm{l} / \mathrm{h}^{-1}\right)$ for 6 hours (CLP $6 \mathrm{~h}+$ TEA). In the hypodynamic sepsis group, normal saline $(15 \mu \mathrm{l} /$ $h^{-1}$ ) was continuously infused through the epidural catheter for 24 hours (CLP $24 \mathrm{~h}$ ). The TEA-treated hypodynamic sepsis group received a continuous epidural infusion of isobaric bupivacaine $0.5 \%\left(15 \mu \mathrm{l} / \mathrm{h}^{-1}\right)$ for 24 hours after injury (CLP $24 \mathrm{~h}+$ TEA). Concentrations of bupivacaine were used as described previously $[10,13]$.

Six and 24 hours after sham laparotomy or induction of peritonitis, respectively, mean arterial pressure (MAP), heart rate (HR), and respiratory rate were measured, and blood was withdrawn from the arterial catheter in awake, spontaneously breathing rats. Arterial hemoglobin, hematocrit, $\mathrm{pH}, \mathrm{paO}_{2}$, $\mathrm{paCO}_{2}, \mathrm{HCO}_{3}{ }^{-}$, actual base excess (ABE), leukocytes, and arterial serum lactate concentrations were determined with blood gas analysis (ABL, 620 Radiometer, Copenhagen, Denmark). 


\section{Isolated perfused lungs}

After hemodynamic measurements and blood gas analysis, rats were again anesthetized with intraperitoneally injected $\alpha$ chloralose $\left(50 \mathrm{mg} / \mathrm{kg}^{-1}\right.$; Sigma Chemicals, Deisenhofen, Germany) and urethane (650 mg/ $\mathrm{kg}^{-1}$, Sigma Chemicals) and were then tracheostomized with a 17-gauge cannula. The lungs were mechanically ventilated with warmed $\left(35^{\circ} \mathrm{C}\right)$ and humidified $21 \% \mathrm{O}_{2}, 5 \% \mathrm{CO}_{2}$, and balanced $\mathrm{N}_{2}$ by using a rodent ventilator (tidal volume, $1 \mathrm{ml} / 100 \mathrm{~g}^{-1}$; frequency, 60 breaths/min ${ }^{-1}$; Harvard Apparatus $\mathrm{GmbH}$, Deisenhofen, Germany). End-expiratory pressure was set at $1 \mathrm{~mm} \mathrm{Hg}$.

For isolated lung perfusion, a sternotomy was conducted, followed by excision of sections of the right and left anterior chest wall to expose heart and lungs. After heparinization (100 $U$ ), rats were partly exsanguinated by needle aspiration (5 to 6 $\mathrm{ml}$ ). A steel cannula (13-gauge) connected to the perfusion system was inserted through the pulmonic valve into the main pulmonary artery by incision in the right ventricle. A suture tied around the pulmonary artery and aorta secured the cannula. The latter prevented systemic blood flow. The circle was closed by a cannula ( $3.5 \mathrm{~mm}$ outside diameter) that was inserted through the apex of the left ventricle and secured with umbilical tape. The isolation and perfusion procedure strictly followed an established and standardized technique $[10,17]$.

The perfusate consisted of the rat's own blood (added to the perfusate after lung isolation) diluted with physiologic salt solution (containing in $\mathrm{mmol} / \mathrm{L}: 119.0 \mathrm{NaCl}, 4.7 \mathrm{KCl}, 1.17$ $\mathrm{MgSO}_{4} \times 7 \mathrm{H}_{2} \mathrm{O}, 22.6 \mathrm{NaHCO}_{3}, 1.18 \mathrm{KH}_{2} \mathrm{PO}_{4}, 3.2 \times 2 \mathrm{H}_{2} \mathrm{O}$, 5.6 dextrose) to a hematocrit of $9 \%$ to $12 \%$. Indomethacin (30 $\mu \mathrm{g} / \mathrm{ml}^{-1}$ ) was added to block prostaglandin synthesis. The perfusate drained from the left ventricle to a glass reservoir and was heated to $38^{\circ} \mathrm{C}$ by a circumferential water jacket. With a peristaltic pump (Harvard Apparatus), the perfusate was returned to the pulmonary artery at constant flow $\left(16 \mathrm{ml} / \mathrm{min}^{-}\right.$ 1). The isolated lung preparation remained in the thoracic cavity lying supine and was warmed with a heating lamp. A warmed and humidified chamber was placed over the thoracic cavity to maintain thoracic temperature at $37^{\circ} \mathrm{C}$. Reservoir $\mathrm{pH}$ was continuously monitored (Hanna Instruments) and maintained at 7.35 to 7.45 by addition of $\mathrm{HCl}$ or $\mathrm{NaOH}$, as required. Pulmonary artery pressure $\left(P_{a}\right)$ was continuously monitored by using a pressure transducer (Becton Dickinson, Singapore). Mean pulmonary venous pressure was set at 2 $\mathrm{mm} \mathrm{Hg}$ by adjusting the height of the reservoir and was held constant. Normoxic $\left(21 \% \mathrm{O}_{2}\right)$ and hypoxic $\left(3 \% \mathrm{O}_{2}\right)$ gas mixtures were administered through individual flowmeters. The inspired $\mathrm{O}_{2}$ concentration was monitored (Oxydig, Draeger, Germany) near the tracheal tube. The model of the isolated and perfused lungs is illustrated in Figure 1. After thoracotomy and stabilization of isolation and perfusion of the lung, receptor-mediated vasoconstriction was studied, as described later.
Figure 1

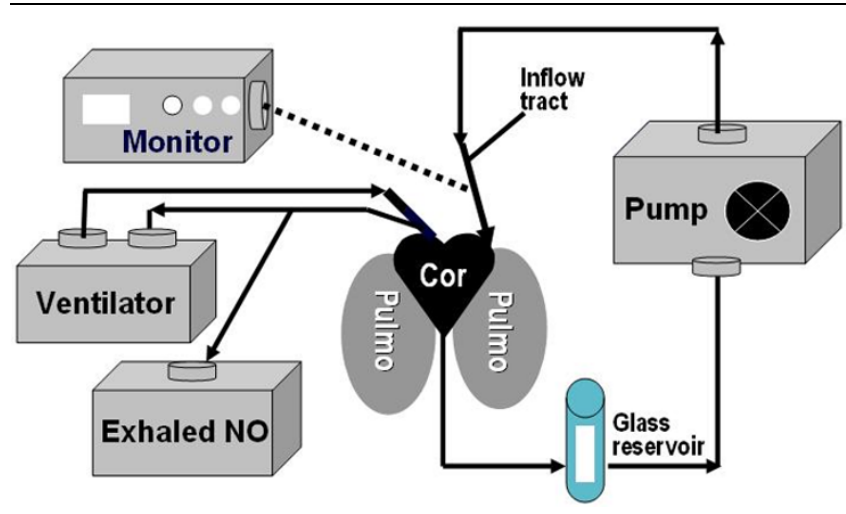

Illustration of the isolated and perfused lung model. After anesthesia and tracheostomy, exhaled NO (exNO) from rat's lungs was measured, followed by isolation and perfusion of the pulmonary circuit. Angiotensin II (Ang II) was injected into the inflow tract of the circuit to test vasoreactivity. Changes in perfusion pressure $(\Delta p)$ from baseline were measured in millimeters of mercury. After a stabilization phase, bradykinin (BK)-induced pulmonary vasoconstriction was induced. Therefore, increasing concentrations of BK $(1,3$, and $6 \mu \mathrm{g})$ were injected into the inflow tract. Changes in perfusion pressure were measured in $\mathrm{mm} \mathrm{Hg}$ and expressed as $\Delta \mathrm{p}$ from baseline pressure.

\section{Exhaled NO}

Exhaled NO (exNO) was used as a surrogate marker for pulmonary NO production [18]. Before the isolation and perfusion of the lungs, the exhaled air from the rats' lungs was collected in a bag for 10 minutes. The exNO concentration was analyzed with a TE $42 \mathrm{~S} \mathrm{NO}_{\mathrm{x}}$ analyzer (Thermo Environment, Franklin, MA, USA) by using an established protocol [10].

\section{Receptor-dependent pulmonary vasoconstriction}

Integrity of vascular smooth muscle cell (VSMC) function is critical for the regulation of the vascular tone. In this study, the potent receptor-mediated vasoconstrictor angiotensin II (Ang II, $0.1 \mu \mathrm{g}$; Sigma Chemicals) was injected into the inflow tract of the isolated perfused lung circuit to test possible functional impairment of VSMCs caused by sympathetic blockade or sepsis. Changes in perfusion pressure were measured in millimeters of mercury and expressed as pressure difference $(\Delta p)$ from baseline pressure $[10,17,19]$.

To test pulmonary endothelial dysfunction, bradykinin (BK)induced vasoconstriction was evaluated by injecting increasing concentrations of BK (1, 3, and $6 \mu \mathrm{g}$; Sigma Chemicals) into the inflow circuit. Each dose was administered 5 minutes after the perfusion pressure returned to baseline levels. Changes in perfusion pressure were measured in millimetres of mercury and expressed as $\Delta \mathrm{p}$ from baseline pressure $[11,17,20]$. 


\section{Myeloperoxidase (MPO) activity}

After the experiment, right lungs were immediately frozen and stored at $-70^{\circ} \mathrm{C}$ until use. MPO, indicating pulmonary neutrophil infiltration, was measured in equal-sized samples of the right lungs by using an established protocol [21]. Results were expressed as microunits of MPO per milligram of protein of supernatant, as determined by bicinchoninic acid assay (Pierce Chemical Co., Rockford, IL, USA).

\section{Pulmonary wet- to dry-weight ratio}

Pulmonary bloodless wet- to dry-weight ratio was determined at the end of the experiment. The left bronchus was carefully separated from the right bronchus and tied, and the left lung was severed. The lung was weighted immediately and dried at $60^{\circ} \mathrm{C}$ in an oven for 4 days to determine the ratio of wet-lung weight (WW) to dry-lung weight (DW). The dry-lung weight became stable after 48 hours of desiccation in our study [10].

\section{Statistical analysis}

Sigma Stat. 3.1 (Systat Software GmbH, Erkrath, Germany) software was used for statistical analysis. After confirming normal distribution of all values (Kolmogorov-Smimov test), a twoway analysis of variance for repeated measurements was performed to detect potential differences within and between groups. In case of significant group differences, post hoc comparisons were made by using the Student-Newman-Keuls test. Bradykinin-induced vasoconstriction was expressed as the peak $P_{a}$ minus baseline $P_{a}$. For all statistical tests, significance was assumed when $P$ was less than 0.05. Data are presented as mean \pm SEM.

\section{Results \\ Systemic and pulmonary effects in healthy animals and healthy animals with TEA}

Healthy animals (SHAM) exhibited stable HR and MAP, which were not affected by the use of TEA (SHAM + TEA). Leukocyte count was higher in healthy animals treated with TEA than in SHAM animals without TEA $(P<0.05)$. Arterial blood gases were similar between SHAM and SHAM + TEA animals (Tables 1 and 2).

Pulmonary MPO activity and exNO levels did not differ between SHAM + TEA and SHAM (Figure 2 and Table 1). Pulmonary endothelial integrity was maintained as indicated by the absence of bradykinin-induced pulmonary vasoconstriction (Figure 3). In addition the pulmonary wet/dry-weight ratio and myeloperoxidase activity remained unchanged under the treatment with TEA (Table 1).

VSMC function in SHAM + TEA animals, as indicated by unchanged Ang Il-induced pulmonary vasoconstriction was not different from that in SHAM (Figure 4).

\section{Systemic and pulmonary effects of TEA in hyperdynamic sepsis}

In hyperdynamic sepsis (6 hours after injury), MAP and HR remained unchanged in untreated (CLP $6 \mathrm{~h}$ ) and TEA-treated septic rats (CLP $6 \mathrm{~h}+$ TEA) when compared with SHAM (Table 1). CLP $6 \mathrm{~h}+$ TEA animals had a significantly lower respiratory rate than did hyperdynamic rats without treatment and SHAM animals $(P<0.05$ versus CLP $6 \mathrm{~h}+$ TEA and SHAM; Table 1). Leukocyte count was significantly reduced in animals with sympathetic blockade when compared with the SHAM

Table 1

\begin{tabular}{|c|c|c|c|c|c|c|}
\hline Variable & SHAM & SHAM + TEA & CLP $6 \mathrm{~h}$ & CLP 6 h + TEA & CLP $24 \mathrm{~h}$ & CLP $24 \mathrm{~h}+$ TEA \\
\hline $\begin{array}{l}\mathrm{HR} \\
\text { (beats/min) }\end{array}$ & $396 \pm 20$ & $412 \pm 24$ & $387 \pm 23$ & $412 \pm 30$ & $464 \pm 16^{\# \star}$ & $442 \pm 37$ \\
\hline $\begin{array}{l}\text { MAD } \\
(\mathrm{mm} \mathrm{Hg})\end{array}$ & $139 \pm 3$ & $130 \pm 9$ & $129 \pm 8$ & $145 \pm 5$ & $133 \pm 3$ & $126 \pm 6$ \\
\hline $\begin{array}{l}\mathrm{RR} \\
\text { (rate/minute) }\end{array}$ & $107 \pm 8$ & $95 \pm 9$ & $94 \pm 9$ & $74 \pm 6^{\#}$ & $130 \pm 7^{\# \star}$ & $115 \pm 5$ \\
\hline Lactate $(\mathrm{mmol} / \mathrm{dl})$ & $\begin{array}{l}0.95 \\
(0.9 / 1.2)\end{array}$ & $\begin{array}{l}1.1 \\
(1.0 / 1.53)\end{array}$ & $\begin{array}{l}1.25 \\
(1.1 / 1.45)\end{array}$ & $\begin{array}{l}1.1 \\
(0.93 / 1.4)\end{array}$ & $\begin{array}{l}2.3^{\# *} \\
(1.83 / 3.1)\end{array}$ & $\begin{array}{l}1.4 \S \\
(1.3 / 1.8)\end{array}$ \\
\hline $\begin{array}{l}\text { Leukocytes } \\
(1,000 / \mu \mathrm{l})\end{array}$ & $5,217 \pm 309$ & $7,184 \pm 552^{\#}$ & $3,868 \pm 878$ & $3,194 \pm 771^{\#}$ & $2,076 \pm 343^{\# \star}$ & $1,752 \pm 552^{\#}$ \\
\hline $\begin{array}{l}\mathrm{Hb} \\
(\mathrm{g} / \mathrm{dl})\end{array}$ & $15.6 \pm 0.5$ & $14.8 \pm 0.2$ & $15.3 \pm 0.7$ & $14.3 \pm 1.2$ & $14.9 \pm 0.2$ & $14.4 \pm 1.0$ \\
\hline MPO ( $\mu \mathrm{U} / \mathrm{mg}$ protein) & $\begin{array}{l}0.48 \\
(0.42 / 0.57)\end{array}$ & $\begin{array}{l}0.47 \\
(0.42 / 0.81)\end{array}$ & $\begin{array}{l}0.92 \\
(0.87 / 1.08)^{\#}\end{array}$ & $\begin{array}{l}0.97 \\
(0.83 / 1.1)^{\#}\end{array}$ & $\begin{array}{l}2.0 \\
(1.91 / 2.17)^{\#}\end{array}$ & $\begin{array}{l}0.26 \\
(0.2 / 1.4)^{\S}\end{array}$ \\
\hline W/D & $4 \pm 1.4$ & $4.4 \pm 0.2$ & $5.5 \pm 0.2^{\#}$ & $5.4 \pm 0.3^{\#}$ & $5.5 \pm 0.2^{\#}$ & $5.5 \pm 0.1^{\#}$ \\
\hline
\end{tabular}

Measurements were performed in awake rats. $\mathrm{HR}=$ heart rate; $\mathrm{MAD}=$ mean arterial pressure; RR = respiratory rate. Data are expressed as mean \pm SEM or median, $25 \%$, and $75 \%$ percentile. $\# P<0.05$ versus SHAM; $+\mathrm{P}<0.05$ versus all CLP groups, $\$$ P $<0.05$ versus same group without TEA; * $\mathrm{P}<0.05$ versus hyperdynamic sepsis (CLP $6 \mathrm{~h}$ ). 
Table 2

Arterial blood gas analysis of the different groups in awake rats spontaneously breathing room air

\begin{tabular}{lllllll}
\hline Variable & SHAM & SHAM + TEA & CLP $6 \mathrm{~h}$ & CLP $6 \mathrm{~h}+$ TEA & CLP 24 h & CLP 24 h + TEA \\
\hline $\mathrm{pH}$ & $7.44 \pm 0.01$ & $7.47 \pm 0.02$ & $7.42 \pm 0$ & $7.42 \pm 0.01$ & $7.44 \pm 0$ & $7.36 \pm 0.03^{\# \S}$ \\
$\mathrm{PaCO}_{2}$ & $38 \pm 0$ & $38 \pm 0$ & $40 \pm 0$ & $41 \pm 2$ & $37 \pm 0$ & $47 \pm 22^{\# \S}$ \\
$\mathrm{PaO}_{2}$ & $97 \pm 2$ & $92 \pm 3$ & $100 \pm 2$ & $100 \pm 5$ & $93 \pm 2$ & $86 \pm 6^{\#}$ \\
$\mathrm{HCO}^{3-}$ & $24.8 \pm 0.3$ & $26.9 \pm 1.2$ & $25.3 \pm 0.3$ & $26.3 \pm 0.9$ & $25.8 \pm 1.3$ & $24.9 \pm 1$ \\
$\mathrm{ABE}$ & $1.0 \pm 1$ & $3.5 \pm 1$ & $1.3 \pm 0$ & $2.3 \pm 1$ & $1.5 \pm 0.5$ & $-0.4 \pm 1.4$ \\
\hline
\end{tabular}

Data are expressed as median \pm SEM. ${ }^{*} P<0.05$ versus SHAM; $\$ P<0.05$ versus same group without TEA. ABE $=$ actual base excess.

group ( $P<0.05$ versus SHAM; Table 1$)$. No changes in pulmonary gas exchange were detected between the two hyperdynamic sepsis groups and SHAM animals (Table 2).

TEA prevented the increase of exNO concentrations in hyperdynamic sepsis, which was comparable to levels of healthy animals $(P<0.05$ versus CLP 6 h; Figure 2). In contrast, MPO activity remained elevated, independent of sympathetic blockade $(P<0.05$ versus SHAM; Table 1$)$. The development of pulmonary endothelial dysfunction was attenuated under the treatment with TEA, as indicated by reduced bradykinininduced pulmonary vasoconstriction $(P<0.05$ versus CLP 6 $\mathrm{h}$; Figure 3). Pulmonary edema remained increased in CLP $6 \mathrm{~h}$ and CLP $6 \mathrm{~h}+$ TEA $(P<0.05$ versus SHAM; Table 1$)$.

Ang II-induced pulmonary vasoconstriction remained unchanged in rats with hyperdynamic sepsis with and without

\section{Figure 2}

\section{Bradykinin-induced pulmonary vasoconstriction}

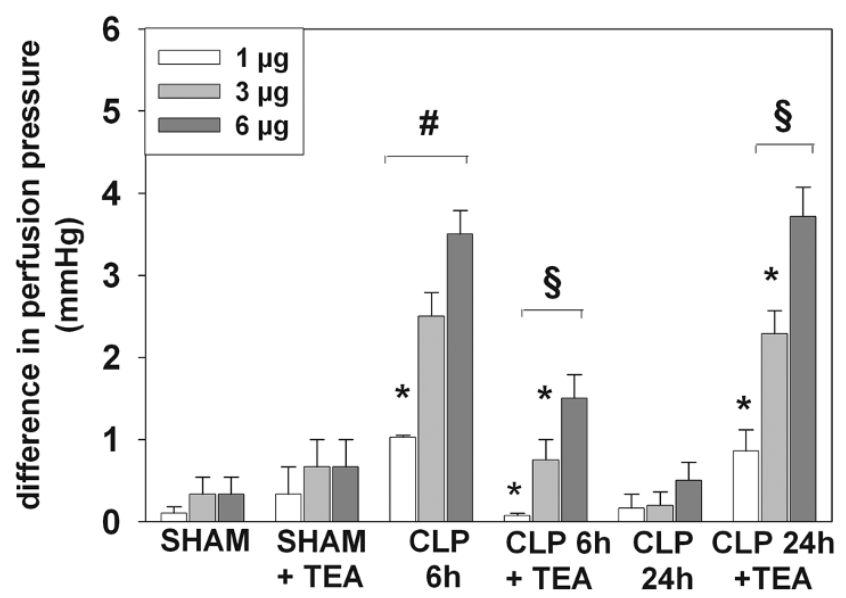

Exhaled NO (parts per billion) in sham animals, early and late sepsis, when compared with sham and septic animals treated with TEA (bupivacaine $0.5 \% ; n=6$ in each group). $\# P<0.05$ versus $\mathrm{SHAM}$; $\mathrm{P}<<$ 0.05 versus groups without TEA. Data are expressed as mean $\pm \mathrm{SEM}$. sympathetic blockade and was comparable to those of SHAM animals (Figure 4).

\section{Systemic and pulmonary effects of TEA in hypodynamic sepsis}

In hypodynamic sepsis (24 hours after injury), untreated animals (CLP $24 \mathrm{~h}$ ) developed profound tachycardia, tachypnea, leukopenia, and increased serum-lactate levels when compared with healthy animals and with hyperdynamic sepsis (all $P$ values $<0.05$ vs. SHAM and CLP 6 h; Table 1). Pulmonary gas exchange in the CLP $24 \mathrm{~h}$ group remained unchanged when compared with SHAM (Table 2). Under the treatment with TEA (CLP $24 \mathrm{~h}+$ TEA), hemodynamic variables did not differ from those in healthy animals, and serum-lactate levels were lower than those in untreated animals with hypodynamic

\section{Figure 3}

\section{Angiotensin II-induced pulmonary vasoconstriction}

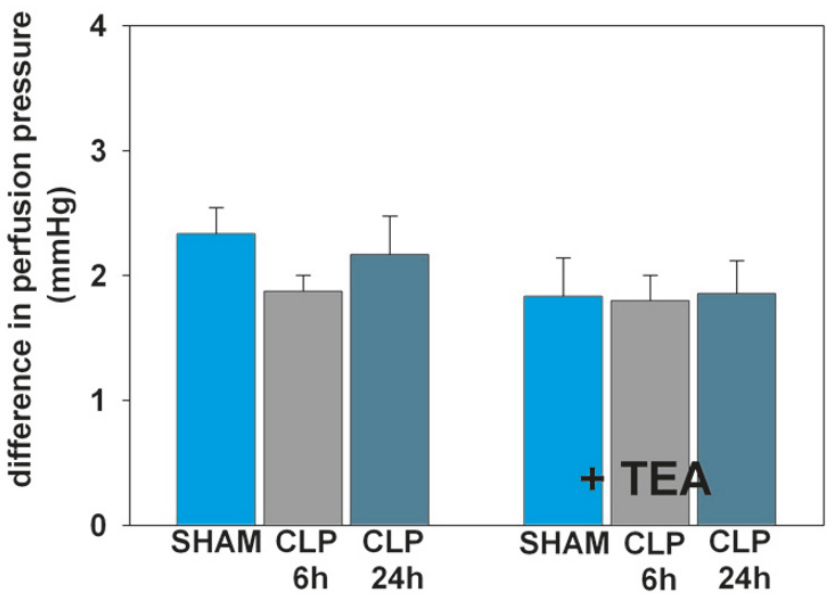

Comparison of bradykinin (BK)-induced vasoconstriction in isolated perfused rat lungs for evaluation of endothelial dysfunction $(n=6$ in each group). The difference in perfusion pressure due to different concentrations of BK $(1,3$, and $6 \mu \mathrm{g})$ is demonstrated. Data are presented as mean \pm SEM. ${ }^{\#} P<0.05$ versus SHAM; $§ P<0.05$ versus the same group without TEA; * $P<0.05$ versus $B K, 6 \mu \mathrm{g}$, within group. 
Figure 4

\section{exhaled NO}

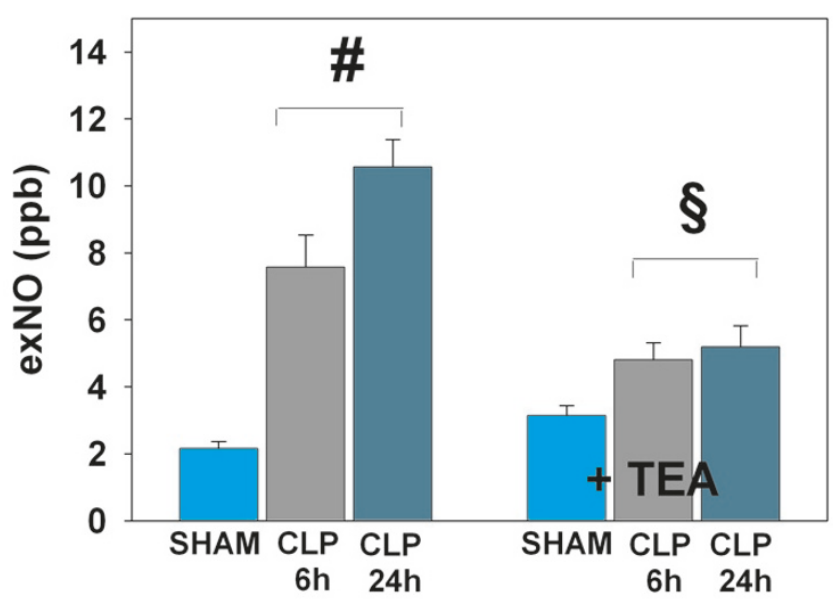

Angiotensin II-induced pulmonary vasoconstriction to test pulmonary smooth muscle cell function in healthy animals, early sepsis (CLP $6 \mathrm{~h}$ ) and late sepsis (CLP $24 \mathrm{~h}$ ) compared with groups receiving treatment with thoracic epidural anesthesia (TEA, $n=6$ in each group). Data are expressed as mean \pm SEM.

sepsis ( $P<0.05$ versus CLP 24 h; Table 1 ). Arterial blood gas analysis revealed a decrease in $\mathrm{PaO}_{2}$ values when compared those in healthy animals ( $P<0.05$ versus SHAM) and an increase in $\mathrm{paCO}_{2}$ in TEA-treated rats when compared with CLP $24 \mathrm{~h}$ and SHAM $(P<0.05$ versus SHAM and CLP $24 \mathrm{~h}$; Table 2).

Sympathetic blockade reduced exNO and MPO activity below levels seen in the SHAM group $(P<0.05$ versus CLP $24 \mathrm{~h}$; Table 1 and Figure 2) but increased bradykinin-induced pulmonary vasoconstriction in contrast to the CLP $24 \mathrm{~h}$ group ( $P$ $<0.05$ versus CLP $24 \mathrm{~h}$ and SHAM; Figure 3). Pulmonary edema was elevated in both treated and untreated hypodynamic sepsis $(P<0.05$ versus SHAM; Table 1$)$. Ang IIinduced pulmonary vasoconstriction remained unchanged in TEA-treated and untreated hypodynamic sepsis and were comparable to the results of the SHAM group (Figure 4).

\section{Discussion}

In the present study, the impact of a regional thoracic sympathetic blockade on pulmonary endothelial function was investigated in both hyperdynamic and hypodynamic sepsis by using an established rodent CLP model $[11,13,17]$. The major findings were (a) that implementation of TEA in hyperdynamic sepsis reduced pulmonary endothelial dysfunction, whereas sympathetic blockade in hypodynamic sepsis resulted in a deterioration of endothelial integrity, (b) that treatment with TEA exhibited antiinflammatory effects with modulation of the NO pathway in both hyperdynamic and hypodynamic sepsis, and (c) that TEA reduced neutrophil migration into the lungs only in hypodynamic sepsis.

One of the most established and clinically relevant sepsis models is the CLP procedure followed by fluid resuscitation and analgesia. It is a typical feature of this model that the time course of sepsis and the diversity of the bacterial flora closely mimic the clinical situation [9]. In this context, it is noteworthy that the CLP sepsis model is characterized by a biphasic hemodynamic response. Notably, an early hyperdynamic phase ( 2 to 10 hours after CLP) is followed by a hypodynamic circulatory phase between 10 and 20 hours $[15,16]$. Although several studies already addressed hemodynamic and intestinal effects of epidural anesthesia in CLP sepsis [14], it was still unclear whether (a) TEA has an impact on pulmonary endothelial (dys)function, and (b) the effects of sympathetic blockade on endothelial dysfunction differ in hyperdynamic and hypodynamic sepsis.

One established technique to investigate pulmonary endothelial dysfunction is the measurement of bradykinin-induced pulmonary vasoconstriction in isolated and perfused rat lungs $[11,17]$. In this context, Fischer and colleagues [11,17] demonstrated that administration of bradykinin into the pulmonary circuit results in a paradoxic vasoconstriction through $\mathrm{BK}_{2}$ receptors when the endothelial layer is injured after a septic insult $[11,17]$. In contrast, healthy control lungs with an intact endothelial layer lacked this bradykinin-induced vasoconstriction. In accordance with the observation of Fischer and associates $[11,17,20]$, we also demonstrated that in hyperdynamic sepsis, administration of bradykinin resulted in a paradoxic pulmonary vasoconstriction in the current study. This effect was not seen in healthy animals and in healthy animals treated with TEA, indicating that animals of the two sham groups did not have endothelial injury. However, impairment of pulmonary VSMC function may have been responsible for a reduced vasoconstrictive response to bradykinin in the different groups. In this regard, it recently was reported that pulmonary VSMC function is affected at different stages of sepsis, thereby modulating pulmonary vasoconstriction [22]. Therefore, we decided to test VSCM function to exclude its influence by stimulating the receptor with Ang II as a different second messenger. With the observation of a uniform vasoconstrictive response in the different groups, it is unlikely that an impairment of VSMC function has contributed to the differences of bradykinin-induced pulmonary vasoconstriction among groups.

Pulmonary NO has been identified as a key mediator in the pathogenesis of endothelial dysfunction in both experimental sepsis and the clinical setting [17,23]. In animal sepsis models, selective inhibitors of the inducible NO synthase resulted in decreased exNO levels and significantly attenuated bradykinin-induced pulmonary vasoconstriction $[17,19]$. In this context, it is another finding of our study that TEA exhibited 
antiinflammatory properties by suppressing exNO as a surrogate marker of iNOS activity in both hyperdynamic and hypodynamic sepsis and additionally reduced neutrophil influx into the lungs in the hypodynamic phase. However, this antiinflammatory effect reduced endothelial dysfunction only in the hyperdynamic phase, whereas the impact of sympathetic blockade in hypodynamic sepsis promoted a deterioration of endothelial function. These results were somewhat unexpected, because iNOS generation from neutrophils and alveolar macrophages is known to play a critical role in the development of septic lung injury $[6,24]$. Moreover, Farley and colleagues [25] recently identified iNOS from alveolar macrophages as a key mediator in the development of pulmonary microvascular endothelial cell septic barrier dysfunction. One of the major roles of alveolar macrophages is to facilitate the recruitment of circulating neutrophils to the lungs, through production and release of neutrophil cytokines [26]. In addition, depletion of alveolar macrophages has been shown to attenuate septic neutrophil influx directly in murine septic lung injury [27]. Although not directly measured in our study, the present data suggest that TEA-related attenuation of MPO activity and reduction of exNO levels in the lungs may be dependent on changes of alveolar macrophage activity. This assumption is supported by a study of Fujii and colleagues [6], who demonstrated that inhibition of alveolar macrophages eliminated the increase in lung iNOS activity and protein expression and significantly attenuated the increase in pulmonary exNO product in endotoxemic rats.

The fact that TEA did not exhibit protective properties on endothelial integrity in hypodynamic sepsis despite its antiinflammatory action may be explained by a study of Traeger and colleagues [28], who reported that selective depletion of pulmonary alveolar macrophages increased lung injury in a peritonitis sepsis model by attenuating the defense mechanisms against gut-derived bacteria in the lungs. Our data are further supported by the observation of others that suppression of NO production reduces endothelial dysfunction in hyperdynamic sepsis [17]. However, this is the first study showing that the effects of sympathetic blockade time-dependently change their characteristics with the development of hypodynamic sepsis. In this regard, it is likely that TEA-related suppression of neutrophil migration and NO production reduced the host defense in the lungs, thereby deteriorating endothelial dysfunction [28]. Because we cannot exclude the possibility of negative cardiovascular effects under sympathetic blockade as a trigger for increased pulmonary endothelial dysfunction, future studies are needed to address this issue in more detail. This study has some limitations. First, the characteristic of this isolated perfused rat-lung model is an ex vivo investigation of pulmonary effects. Although this model is an established technique for studies of lung pathology $[17,19,20,29]$ and allows measurements in the pulmonary circuit that are impossible in in vivo settings, further work is needed to confirm our findings in vivo. Second, we cannot exclude effects of systemic absorption of epidurally applied bupivacaine and subsequent antiinflammatory pulmonary effects. It was recently reported that intravenously administered local anesthetics accounted for reduced lung injury in different animals sepsis models $[30,31]$, so further studies are needed to clarify the role of intravenously applied local anesthetics on inflammatory response in the presence of sepsis. Third, this experiment was not carried out to investigate the safety of TEA in critical illness, but to identify the effects of TEA on the pulmonary endothelial integrity. Although the use of TEA exhibited hemodynamic stability in hyperdynamic and hypodynamic sepsis, we are unable to comment on TEA-related actions on other organ systems. In this regard, however, it is noteworthy that the use of TEA in both hypodynamic and hyperdynamic endotoxemic sheep did not impair hemodynamics beyond the changes caused by endotoxin itself $[32,33]$. Although the use of TEA in critically ill patients has gained increasing interest in the recent years, its use in the intensive-care setting remains controversial [2-4]. Therefore, further studies are needed to assess the efficacy and safety of TEA in critical illness.

\section{Conclusions}

In summary, this study demonstrates for the first time that TEA exerts protective effects on pulmonary endothelial integrity only in hyperdynamic sepsis. Whether the negative effects on endothelial function in hypodynamic sepsis have an impact on overall morbidity and mortality remains to be determined in future studies.

\section{Key messages}

- Implementation of TEA reduced pulmonary endothelial dysfunction in hyperdynamic sepsis.

- Sympathetic blockade resulted in a deterioration of endothelial integrity in hypodynamic sepsis.

- Treatment with TEA exhibited antiinflammatory effects with modulation of the NO pathway in both hyperdynamic and hypodynamic sepsis.

- TEA reduced neutrophil migration into the lungs only in hypodynamic sepsis.

\section{Competing interests}

The authors declare that they have no competing interests.

\section{Authors' contributions}

SL contributed to design, funding, data acquisition, and statistical analysis and drafted the manuscript; HF contributed to the design of the study and data acquisition; MW participated in data analysis and manuscript drafting; $A Z$ participated in data acquisition and analysis; MF participated in data acquisition; HVA participated in the design and manuscript drafting; AWS contributed to planning the study and statistical analysis; LGF took part in study design, funding, data acquisition, statistical analysis, and drafting the manuscript. 


\section{Acknowledgements}

The authors thank Christina Grosserichter, Department of Anesthesiology and Intensive Care Medicine, University Hospital Muenster, Germany, for expert technical assistance during the study. The study was financially supported by departmental funding.

\section{References}

1. Clemente A, Carli F: The physiological effects of thoracic epidural anesthesia and analgesia on the cardiovascular, respiratory and gastrointestinal systems. Minerva Anestesiol 2008, 74:549-563.

2. Low JH: Survey of epidural analgesia management in general intensive care units in England. Acta Anaesthesiol Scand 2002, 46:799-805.

3. Spackman DR, McLeod AD, Prineas SN, Leach RM, Reynolds F: Effect of epidural blockade on indicators of splanchnic perfusion and gut function in critically ill patients with peritonitis: a randomised comparison of epidural bupivacaine with systemic morphine. Intensive Care Med 2000, 26:1638-1645.

4. Sielenkamper AW, Van Aken $\mathrm{H}$ : Thoracic epidural anesthesia: more than just anesthesia/analgesia. Anesthesiology 2003, 99:523-525.

5. Ware LB, Matthay MA: The acute respiratory distress syndrome. N Engl J Med 2000, 342:1334-1349.

6. Fujii $Y$, Goldberg P, Hussain SN: Contribution of macrophages to pulmonary nitric oxide production in septic shock. Am J Respir Crit Care Med 1998, 157:1645-1651.

7. Lee RP, Wang D, Kao SJ, Chen HI: The lung is the major site that produces nitric oxide to induce acute pulmonary oedema in endotoxin shock. Clin Exp Pharmacol Physiol 2001, 28:315-320.

8. Popping DM, Elia N, Marret E, Remy C, Tramer MR: Protective effects of epidural analgesia on pulmonary complications after abdominal and thoracic surgery: a meta-analysis. Arch Surg 2008, 143:990-999. discussion 1000

9. Wichterman KA, Baue $\mathrm{AE}$, Chaudry $\mathrm{IH}$ : Sepsis and septic shock: a review of laboratory models and a proposal. I Surg Res 1980, 29:189-201.

10. Lauer S, Freise H, Fischer LG, Singbartl K, Aken HV, Lerch MM, Sielenkamper AW: The role of thoracic epidural analgesia in receptor-dependent and receptor-independent pulmonary vasoconstriction in experimental pancreatitis. Anesth Analg 2007, 105:453-459.

11. Fischer LG, Horstman DJ, Hahnenkamp K, Kechner NE, Rich GF: Selective iNOS inhibition attenuates acetylcholine- and bradykinin-induced vasoconstriction in lipopolysaccharide-exposed rat lungs. Anesthesiology 1999, 91:1724-1732.

12. Freise H, Anthonsen S, Fischer LG, Van Aken HK, Sielenkamper AW: Continuous thoracic epidural anesthesia induces segmental sympathetic block in the awake rat. Anesth Analg 2005, 100:255-262.

13. Freise H, Lauer S, Anthonsen S, Hlouschek V, Minin E, Fischer LG, Lerch MM, Van Aken HK, Sielenkamper AW: Thoracic epidural analgesia augments ileal mucosal capillary perfusion and improves survival in severe acute pancreatitis in rats. Anesthesiology 2006, 105:354-359.

14. Daudel F, Freise H, Westphal M, Stubbe HD, Lauer S, Bone HG, Aken HV Sielenkamper AW: Continuous thoracic epidural anesthesia improves gut mucosal microcirculation in rats with sepsis. Shock 2007, 28:610-614.

15. Yang S, Zhou M, Chaudry IH, Wang P: Novel approach to prevent the transition from the hyperdynamic phase to the hypodynamic phase of sepsis: role of adrenomedullin and adrenomedullin binding protein-1. Ann Surg 2002, 236:625-633

16. Wang P, Yoo P, Zhou M, Cioffi WG, Ba ZF, Chaudry IH: Reduction in vascular responsiveness to adrenomedullin during sepsis. J Surg Res 1999, 85:59-65.

17. Fischer LG, Hilpert $J H$, Freise $H$, Wendholt $D$, Van Aken $H$ Sielenkamper AW: Bradykinin-induced pulmonary vasoconstriction is time and inducible nitric oxide synthase dependent in a peritonitis sepsis model. Anesth Analg 2004, 99:864-871.

18. Okamoto I, Abe M, Shibata K, Shimizu N, Sakata N, Katsuragi T, Tanaka K: Evaluating the role of inducible nitric oxide synthase using a novel and selective inducible nitric oxide synthase inhibitor in septic lung injury produced by cecal ligation and puncture. Am J Respir Crit Care Med 2000, 162:716-722.

19. Fischer LG, Freise H, Hilpert JH, Wendholt D, Lauer S, Van Aken $\mathrm{H}$, Sielenkamper AW: Modulation of hypoxic pulmonary vasoconstriction is time and nitric oxide dependent in a peritonitis model of sepsis. Intensive Care Med 2004, 30:1821-1828.

20. Fischer LG, Hollmann MW, Horstman DJ, Rich GF: Cyclooxygenase inhibitors attenuate bradykinin-induced vasoconstriction in septic isolated rat lungs. Anesth Analg 2000, 90:625-631.

21. Singbartl K, Green SA, Ley K: Blocking P-selectin protects from ischemia/reperfusion-induced acute renal failure. FASEB $J$ 2000, 14:48-54.

22. Li S, Fan SX, McKenna TM: Role of nitric oxide in sepsisinduced hyporeactivity in isolated rat lungs. Shock 1996, 5:122-129.

23. Lang JD, McArdle PJ, O'Reilly PJ, Matalon S: Oxidant-antioxidant balance in acute lung injury. Chest 2002, 122:314S-320S.

24. Wheeler AP, Bernard GR: Acute lung injury and the acute respiratory distress syndrome: a clinical review. Lancet 2007, 369:1553-1564.

25. Farley KS, Wang LF, Law C, Mehta S: Alveolar macrophage inducible nitric oxide synthase-dependent pulmonary microvascular endothelial cell septic barrier dysfunction. Microvasc Res 2008, 76:208-216.

26. Mercer-Jones MA, Shrotri MS, Peyton JC, Remick DG, Cheadle WG: Neutrophil sequestration in liver and lung is differentially regulated by $\mathrm{C}-\mathrm{X}-\mathrm{C}$ chemokines during experimental peritonitis. Inflammation 1999, 23:305-319.

27. Farley KS, Wang LF, Razavi HM, Law C, Rohan M, McCormack DG, Mehta S: Effects of macrophage inducible nitric oxide synthase in murine septic lung injury. Am J Physiol Lung Cell Mol Physiol 2006, 290:L1164-L1172.

28. Traeger T, Kessler W, Hilpert A, Mikulcak M, Entleutner M, Koerner $P$, Westerholt A, Cziupka K, van Rooijen N, Heidecke CD, Maier S: Selective depletion of alveolar macrophages in polymicrobial sepsis increases lung injury, bacterial load and mortality but does not affect cytokine release. Respiration 2009, 77:203-213.

29. Spohr F, Cornelissen AJ, Busch C, Gebhard MM, Motsch J, Martin EO, Weimann J: Role of endogenous nitric oxide in endotoxininduced alteration of hypoxic pulmonary vasoconstriction in mice. Am J Physiol Heart Circ Physiol 2005, 289:H823-H831.

30. Nishina K, Mikawa K, Takao Y, Shiga M, Maekawa N, Obara H: Intravenous lidocaine attenuates acute lung injury induced by hydrochloric acid aspiration in rabbits. Anesthesiology 1998, 88:1300-1309.

31. Kiyonari Y, Nishina K, Mikawa K, Maekawa N, Obara H: Lidocaine attenuates acute lung injury induced by a combination of phospholipase A2 and trypsin. Crit Care Med 2000, 28:484-489

32. Daudel F, Bone HG, Traber DL, Stubbe HD, Lettau M, Lange M Scharte M, Van Aken H, Westphal M: Effects of thoracic epidural anesthesia on hemodynamics and global oxygen transport in ovine endotoxemia. Shock 2006, 26:615-619.

33. Daudel F, Ertmer C, Stubbe HD, Lange M, Pulina R, Bone HG, Sielenkamper AW, Van Aken H, Westphal M: Hemodynamic effects of thoracic epidural analgesia in ovine hyperdynamic endotoxemia. Reg Anesth Pain Med 2007, 32:311-316. 